\title{
Hybrid Pixel- and Object-Based Approach to Habitat Condition Monitoring
}

\author{
Geoff SMITH \\ Specto Natura Limited, Cambridge/UK · geoffsmith@specto-natura.co.uk \\ This contribution was double-blind reviewed as extended abstract.
}

\begin{abstract}
The analysis of EO data is generally either pixel- or object-based, but this paper outlines a hybrid approach which maximises the information content of the image at its finest spatial resolution (the pixel) and then analyses this information at the scale of landscape features (objects). This approach is useful where the characteristics within features are important to their assessment and is highly relevant in the current context of habitat legislation which focuses on changes in condition as well as extent. A vital component of the approach is local expert knowledge to improve both the results and the engagement of key user communities in the process. Examples are provided for monitoring a 5 year wetland restoration programme where it was aimed to demonstrate a viable operational workflow.
\end{abstract}

\section{Introduction}

The analysis of EO data is divided generally into two basic approaches. Pixel-based approaches consider each image pixel in relative isolation and attempt to identify the characteristics of the area it represents on the surface using its spectral measurements and sometimes those of immediately adjacent pixels. Although relatively straightforward and computationally simple, the results rarely adequately describe the structure of the landscape and suffer from "salt and pepper" effects caused by system noise, mixed pixels and other uncertainties. However, pixel-based approaches do capture landscape variability at the pixel level and can provide useful descriptions of within feature variability which are particularly useful in semi-natural areas where landscape structure can be considered as a continuous field or fuzzy in nature.

In recent years the drawbacks of pixel-based approached have been addressed by objectbased approaches (BLASCHKE 2010) that attempt to extract the actual landscape features as seen in the image by applying one or more segmentations. The features are often related to real world objects such as habitat patches, fields, woods, lakes etc. For each feature the spectral characteristics (e.g. average and variability), structure (e.g. shape and size) and context (e.g. adjacency to others) can be used to identify them. This structured approach allows features to be aggregated and subdivided to better represent the landscape and rulesets can be developed to encompass complex domain knowledge. However, object-based mapping may not always be the most appropriate approach when studying naturally varying

Jekel, T., Car, A., Strobl, J. \& Griesebner, G. (Eds.) (2013): GI_Forum 2013. Creating the GISociety. (C) Herbert Wichmann Verlag, VDE VERLAG GMBH, Berlin/Offenbach. ISBN 978-3-87907-532-4.

C ÖAW Verlag, Wien. eISBN 978-3-7001-7438-7, doi:10.1553/giscience2013s552. 
systems which may exhibit complex multi-scale patterns of surface characteristics (e.g. vegetation cover) or consist of transition zones such as ecotones.

This paper outlines a hybrid approach which aimed to maximise the information content of the image at its finest spatial resolution (the pixel) and then analyse these results at the scale of meaningful landscape features, such as field boundaries derived from external sources or vegetation patches derived from image segmentation (HoFFMANN et al. 2000). This approach is particularly useful where the characteristics within features such as habitat condition are important. Also, it allows the detection of subtle changes within features over time where the extent of the feature remains relatively constant, for instance the thinning of woodland. The examples reported here were developed to address the user requirements associated with a wetland restoration project in the UK. The users were particularly interested in the within feature variations / changes in wetness and heterogeneity.

\section{Method}

Standard object-based image analysis (OBIA) is suitable for the limited spectral information content and fine spatial detail within aerial photography and similar very high spatial resolution (VHR) datasets.

This approach expands the OBIA concept by employing a simple scene component analysis to extract the spectral information from the data at the pixel level in advance of object-based processing. When dealing with VHR data the pixels, rather than representing a habitat or land cover class of interest, may be sub components (e.g. roof facets in buildings). Therefore an unsupervised clustering is performed using the ISODATA algorithm, to maximise the information extracted from the image without imposing an unrealistic nomenclature on the results. The clusters generated are then labelled as scene components which best represent their character using local domain expertise (habitat experts/field ecologists), visual interpretation and comparisons with ground data. The scene component image shows different land cover types or habitats present with different proportions and patterns of scene components. The challenge is therefore to capture these proportions and patterns within a realistic landscape structure through features obtained from existing sources or image segmentation. A set of OBIA tools are used to summarise the scene components on each feature such as proportions, levels of variability, patterns/texture and thematic, spatial or change related indicators. For instance, wetness and heterogeneity of the surface within each feature could be important. The wetness of a feature can be defined as the proportion of the 'wet' scene components while the heterogeneity can be estimated from a measure of relative entropy. From this rich attribute set rules can be developed to identify features in a similar way to conventional OBIA.

\section{Study Site}

The study site for this work was at Wicken Fen in Cambridgeshire, UK, which is an important protected wetland site and part of a major habitat restoration scheme. It is one of only four wild fens which still survive in the enormous Great Fen Basin area of East Anglia and includes fenland, farmland, marsh and reedbeds. The work described here uses false 
colour infrared (FCIR) aerial photography (AP) from 2007 and 2012 resampled to $2 \mathrm{~m}$ spatial resolution within a spatial framework of features digitised from field-surveyed management plans and visual AP interpretation.

\section{Results}

At Wicken Fen the scene components for 2007 and 2012 FCIR AP images were derived using a maximum of 80 clusters and labelled as scene components (Fig. 1). The more intensively managed areas or those that have only recently come into the restoration process are more uniform. Those that are extensively managed or inundated at intervals to recreate fen habitats are more heterogeneous. The scene component images can be used visually by land managers to assess and monitor the impact of policies over large areas.

a)

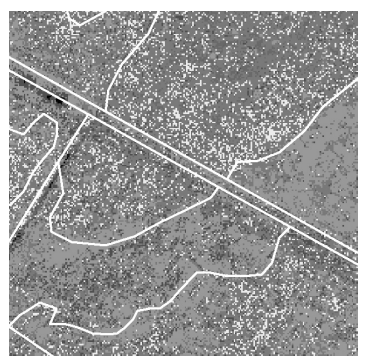

b)

\begin{tabular}{|c|c|c|c|}
\hline No. & Code & Name & Colour \\
\hline 1 & BG & Bare ground & \\
\hline 2 & OW & Open water & \\
\hline 3 & SW & Seasonally wet vegetation & \\
\hline 4 & TD & Tall dense non-woody vegetation & \\
\hline 5 & L & Low dense vegetation late season & \\
\hline 6 & LE & Low dense vegetation early season & \\
\hline 7 & SC & Scrub & \\
\hline 8 & SH & Shadow & \\
\hline 9 & ND & No data & \\
\hline
\end{tabular}

Fig. 1: a) An example of the scene component image with feature objects and b) the scene component legend

To assess the impact of different land management policies the scene components summarised on each feature for each date were compared to identify changes over time. In Fig. 2 a) feature ID 114 has not been impacted that severely by management practices and has remained relatively stable between 2007 and 2012 showing only minor changes due to abandonment. However, in Fig. 2 b) feature ID 170 has been exposed to a controlled inundation policy and there have been significant increases in seasonally wet vegetation (SW) along with smaller increases in bare ground (BG) and open water (OW).

a)

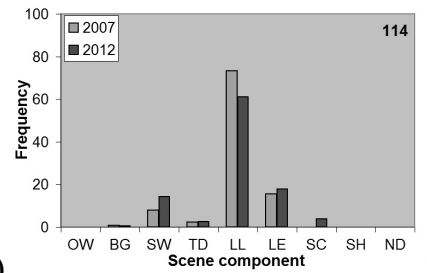

b)

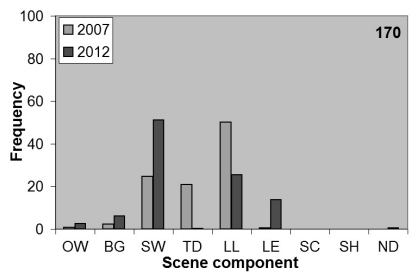

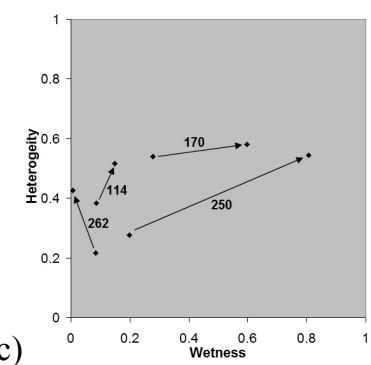

Fig. 2: Examples of a relatively stable (a) and highly dynamic (b) features and an indicator plot of heterogeneity against wetness (c). The three digit numbers refer to the feature ID and the full list of scene component names are given in Fig. 1. 
The wetness and heterogeneity indictors were calculated for each feature on each date to determine if their changes were in line with those proposed by the policy. In Fig. 2 c) the arrows indicate the change from 2007 to 2012 and he specific changes of each feature can be used to assess its particular situation and the likely needs for management changes in the future.

\section{Summary}

The results of this work are currently being fully explored by local domain experts but these initial examinations show that there is useful information held within the scene component images and their summaries the feature level. It is likely that further indicators, especially related to the spatial patterns within the features will be developed to address a range of indicator requirements. Also, more conventional OBIA rule-sets based on the feature attribution are being considered for object identification and categorisation.

The changes identified by this approach may sometimes be known in general terms by the site managers, but these are rarely quantified on a feature by feature basis and compared across the whole site so that changes to policy can be assessed and tested in context. The users at Wicken Fen had specific requirements which this approach addressed and has generated further interest in how remotely sensed datasets can be further exploited within their monitoring programmes. At other sites the changes may require a more conventional object-based approach to map habitat patches as uniform landscape units against a standard nomenclature. The approach described in this paper could be a straight forward add-on to more conventional approaches particularly as habitat condition is now an important part of monitoring to report against national and European regulations.

The approach is not necessarily at the cutting edge of image analysis and GI science, but is designed to be easily adopted by the end user organisation and is thus based on relatively straightforward and established technology. The key aspect is the combination of the technology within expert knowledge into a viable approach that could easily become an operational workflow for the end users or service supplied by a contractor. The interpretative components exploit local expert knowledge which could pull far more value from the dataset than has been possible by a developer and increase the engagement between the landscape ecology and EO communities.

\section{References}

BLASCHKE, T. (2010), Object based image analysis for remote sensing. ISPRS Journal of Photogrammetry and Remote Sensing, 65, 2-16.

HoffmanN, A., Smith, G. M. \& LehMAN, F. (2000), Die Klassifizierung hochauflösender Daten - ein Per-Parcel-Ansatz mit Daten des digitalen Kamerasystems HRSC-A. In: Strobl, J, BlaschKe, T. \& Griesebner, G., Angewandte Geographische Informationsverarbeitung XII. Beiträge zum AGIT-Symposium Salzburg 2000. Heidelberg, Wichmann, 228-237. 